\title{
Regulatory effects of cervical sympathetic trunk and renal sympathetic nerve activities on cerebral blood flow during head-down postural rotations
}

\author{
Noriko Matsuo, Satoshi Matsuo , Yosuke Nakamura, Felix Ojeiru Ezomo, Yasuaki Kawai \\ Division of Adaptation Physiology, Department of Physiology, Tottori University Faculty of Medicine, 86 Nishi-cho, Yonago 683-8503, Japan
}

\section{A R T I C L E I N F O}

\section{Keywords:}

Head-down rotation

Cerebral blood flow

Rat

Cervical sympathetic trunk

Renal sympathetic nerve

\begin{abstract}
A B S T R A C T
This study attempts to clarify the neural control of cerebral blood flow (CBF) during head-down postural rotation, which induces a cephalad fluid shift in urethane-anesthetized rats. The animals were placed on a table, tilted to a $45^{\circ}$ head-down position over $5 \mathrm{~s}$ and maintained in that position. Head-down rotation (HDR) induced a transient decrease $(8 \pm 3 \mathrm{~mm} \mathrm{Hg}$; mean $\pm \mathrm{SE}$ ) in mean arterial blood pressure (ABP) at $7.3 \pm 0.3 \mathrm{~s}$ after the onset of HDR. The pressure returned to the pre-HDR level within $1 \mathrm{~min}$ in the head-down position. Pretreatment with hexamethonium bromide suppressed the HDR-elicited decrease in ABP, suggesting that the decrease in ABP was induced by the suppression of autonomic neural outflow. The administration of phenoxybenzamine (PB), an $\alpha$-adrenergic antagonist, also eliminated the HDR-elicited decrease in ABP, suggesting that this decrease was elicited by the suppression of $\alpha$-adrenergic vascular tone. To test sympathetic outflow during HDR, renal sympathetic nerve activity (RSNA) and cervical sympathetic trunk (CST) activity (CSTA) were recorded. RSNA was transiently suppressed at $2.3 \pm 0.4 \mathrm{~s}$ after HDR onset, followed by a decrease in ABP, suggesting that this decrease was, at least in part, induced by the suppression of sympathetic nerves. CSTA did not change significantly during HDR. These results suggest that HDR suppresses sympathetic nerves in the lower body rather than in the head, which might result in a decrease in $\mathrm{ABP}$. To test the effect of the decrease in ABP due to sympathetic activity on CBF during HDR, changes in CBF during HDR were measured. CBF did not change significantly during HDR in the control group after the administration of an $\alpha$-receptor blocker or after denervation of the CSTs. These results suggest that the impact of the CSTs on CBF is likely to be limited by a rapid increase in CBF due to HDRelicited cephalad fluid shift and that CBF autoregulation proceeds through an alternative mechanism involving the myogenic properties of cerebral vessels.
\end{abstract}

\section{Introduction}

Understanding the mechanisms of orthostatic hypotension and its treatments is necessary to elucidate the neural regulation of blood pressure during postural alterations (Low and Tomalia, 2015). Regarding the neural control of arterial blood pressure (ABP) during postural changes, baroreceptor reflexes (BRs) and the vestibular apparatus are mechanistically important (Doba and Reis, 1974; Eckberg et al., 1976). Many researchers have shown that fluid shift towards the head exerts BRs due to a hydrostatic effect (Guo et al., 1982; Thames et al., 1982; Nagaya et al., 1995; Tanaka et al., 1999; Westerhof et al., 2006; Nakamura et al., 2009). BRs induce inhibition of the renal sympathetic nerve (DiBona and Sawin, 1985). Nakamura et al. (2009) investigated the time course of aortic depressor nerve activity (ADNA), renal sympathetic nerve activity (RSNA), and ABP during head-down rotation (HDR) using anesthetized rabbits. They demonstrated that vestibulo-sympathetic reflexes (VSRs) induced the suppression of RSNA during HDR, which was followed by a transient decrease in ABP. ADNA, the afferent activity of BRs, increased considerably during HDR. Peak ADNA excitation occurred significantly later than peak RSNA suppression, and this suppression was eliminated by vestibular lesions. Thus, this result suggests that the decrease in ABP during HDR is induced by a rapid mechanism that is probably mediated by VSRs (Nakamura et al., 2009).

Why does ABP decrease transiently during HDR? Since a decrease in $\mathrm{ABP}$ reduces cerebral perfusion pressure (CPP), which is the pressure

Abbreviations: CBF, cerebral blood flow; HDR, head-down rotation; ABP, arterial blood pressure; CSTA, cervical sympathetic trunk activity; RSNA, renal sympathetic nerve activity; PB, phenoxybenzamine.

* Corresponding author.

E-mail address: smatsuo@tottori-u.ac.jp (S. Matsuo). 
gradient between systemic blood pressure and intracranial pressure (ICP) (Gierthmühlen et al., 2010; Ainslie and Brassard, 2014; Brassard et al., 2017), the suppression of sympathetic nerves during HDR might operate in a feedforward manner to avoid a rapid increase in cerebral blood flow (CBF) due to a cephalad fluid shift. Therefore, we asked whether this feedforward system suppresses the activity of cervical sympathetic trunks (CSTs) as well as RSNA during HDR. With the level of the heart as a reference, the head moves to a position lower than that of the heart upon HDR by $45^{\circ}$; on the other hand, the animal's lower body moves to a position higher than the heart in HDR. If cephalad fluid shift during HDR elicits the suppression of CST activity (CSTA), this suppression causes a sudden increase in CBF during HDR. This increase in $\mathrm{CBF}$ is not thought to be a pertinent response because hypertensive encephalopathy occurs as the result of a sudden, sustained increase in blood pressure (Cipolla, 2009) and because the increase in CBF might cause brain edema (Kawai et al., 1993; Kawai et al., 1997; Asai et al., 2002). Therefore, to prevent an increase in CBF during HDR, CSTA must be simultaneously activated. Some researchers have observed a certain decrease in CBF at baseline, especially during hypertension upon stimulation of the superior cervical ganglion (Cassaglia et al., 2008; Willie et al., 2014). These researchers have proposed that the sympathetic vasoconstriction of cerebral vessels is a protective mechanism for the brain that limits cerebral perfusion and microcirculatory pressure during transient increases in arterial pressure (Cassaglia et al., 2008). However, CSTA during HDR remains unclear. Thus, we sought to clarify this regional difference in the activities of CSTs and renal sympathetic nerves to elucidate the regulation of $\mathrm{CBF}$ during postural changes.

$\mathrm{CBF}$ autoregulation is the ability of the brain to maintain relatively constant blood flow despite changes in perfusion pressure (Cipolla, 2009). Gotoh et al. (1971) demonstrated a loss of CBF autoregulation in patients with severe impairment of the autonomic nervous system during head-up tilt, suggesting that the autonomic nervous system contributes to autoregulation during postural alteration (Gotoh et al., 1971). Although cerebral autoregulation is widely accepted, the interaction between CSTA and autoregulation remains unclear. Additionally, whether sympathetic outflow affects CBF remains controversial (Strandgaard and Sigurdsson, 2008; van Lieshout and Secher, 2008; Ainslie and Brassard, 2014; Brassard et al., 2017). Thus, if CSTs are activated by HDR, the CSTs are an important candidate for cerebral autoregulation. If not, an alternative mechanism for cerebral autoregulation should be considered. The purpose of this study was to investigate whether HDR affects sympathetic outflow with regional differences. We hypothesized that CSTA does not decrease significantly to prevent a rapid increase in CBF due to a HDR-elicited cephalad fluid shift. This study provides important information to clarify the role of the CST in controlling brain circulation during postural alterations.

\section{Materials and methods}

\subsection{Animal preparation}

Four experiments were performed on 32 Sprague-Dawley rats weighing 350-470 g. In the first experiment (Experiment 1), the RSNA and $\mathrm{ABP}$ of 8 rats were recorded. In the second experiment (Experiment 2 ), the CSTA instead of RSNA and ABP of 8 rats were recorded. In the third experiment (Experiment 3), the $\mathrm{CBF}$ and $\mathrm{ABP}$ of 8 rats whose CSTs were intact were recorded. In the last experiment (Experiment 4), the $\mathrm{CBF}$ and $\mathrm{ABP}$ of 8 rats whose CSTs were bilaterally denervated were measured. All surgical and experimental protocols had been approved by the Animal Care Committee of Tottori University (No: 17-Y-15 and No: 19-Y-11) and complied with the Institute for Laboratory Animal Research Guide for the Care and Use of Laboratory Animals. The animals inhaled room air with 4\% sevoflurane (Abbott Japan Co., Ltd., Tokyo) for the introduction of anesthesia (Murakami et al., 2014). Depths of anesthesia were maintained during surgery with 2 to $3.5 \%$ sevoflurane. The state of anesthesia was monitored by heart rate and blood pressure, which remained stable (Nakamura et al., 2009). Lidocaine (0.5\%) was used for local infiltration anesthesia as necessary. After surgery, the animals were anesthetized via an intraperitoneal injection of urethane $(1.2-1.9 \mathrm{~g} / \mathrm{kg}$, Wako, Tokyo, Japan) during the experimental protocols. A supplementary dose $(0.3-0.5 \mathrm{~g} / \mathrm{kg})$ was added as necessary.

The right femoral artery was cannulated to monitor ABP. A pressure transducer (DT-4812, Viggo-Spectramed, FL) was attached to the thorax of the animal and fixed to maintain a level relative to the heart during HDR. The right femoral vein was also cannulated for intravenous injection.

Regional CBF on the surface of the parietal lobe in rats in the prone position was measured by means of a laser Doppler flowmeter (ALF 21D Laser Doppler Flowmeter, Advance, Tokyo, Japan). After skin incision, a small hole (approximately $2 \mathrm{~mm}$ in diameter) was made on the right parietal skull bone $1.5 \mathrm{~mm}$ lateral to the midline, from which a guide cannula to fix a Doppler probe (1.0 $\mathrm{mm}$ in diameter) was inserted into the parietal lobe. The tip of the probe was placed into the lobe at a depth of $0.5 \mathrm{~mm}$ from the surface and fixed to an anchor attached to the skull bone using dental resin.

To record the sympathetic nerve outflow activity, the right CST and renal sympathetic nerves were exposed. The method used to identify the right renal nerves has been described elsewhere (Nakamura et al., 2009). The CST was isolated at the rostral level of the superior cervical ganglion and separated from the aortic and vagus nerves. A pair of stainless-steel wire electrodes ( $50 \mu \mathrm{m}$ in diameter, California Fine Wire, Grover Beach, CA, USA) insulated with a silicon sheath was used to record each nerve. The tip of each wire was uncovered from the sheath, and the tips were attached to the nerve at a distance of approximately 1 to $1.5 \mathrm{~mm}$ (Nakamura et al., 2009). Then, the area where the wires were attached and space between the nerves were embedded with silicone gel. In Experiment 4, the CSTs were cut bilaterally at the rostral level of the ganglion. A sham operation was carried out for all groups, that is, the bilateral CSTs, unilateral renal nerve, and unilateral surface of the parietal lobe were exposed. Then, all layers of muscle and skin for all incisions were sutured, and the hole on the skull bone was closed with dental resin.

\subsection{Protocols}

After surgical preparation, the animal was placed on a tilting table in the prone position. A potentiometer was placed (Matsuo et al., 1994). After stable ABP had been confirmed, the animal was kept in the prone position for $1 \mathrm{~min}$. Then, the animal was rotated to a $45^{\circ}$ head-down tilted (HDT) position over $5 \mathrm{~s}$ (HDR). The HDT position was maintained for $1 \mathrm{~min}$, after which the animal was rotated back to the horizontal position. HDR was repeated at least 2 times. In Experiment 1, hexamethonium bromide, a ganglionic transmission blocker, was intravenously injected ( $\mathrm{n}=4$, total dose; $15 \mathrm{mg} / \mathrm{kg}$ ) after the HDR protocols, after which HDR was performed two times. In Experiment 3, phenoxybenzamine (PB), an $\alpha$-adrenergic antagonist, was administered via bolus injection within $1 \mathrm{~min}$ (total dose; $0.5 \mathrm{mg} / \mathrm{kg}$ ) after the HDR protocol, after which HDR was performed two times.

\subsection{Measurement and recording}

A Doppler probe inserted into the brain matter was connected to a laser Doppler flow meter, and the blood flow volume was expressed by digit numbers $(\mathrm{ml} / \mathrm{min} / 100 \mathrm{~g})$, which were recorded as a DC voltage. The bipolar wire electrodes were connected to a differential amplifier during Experiment 1 and Experiment 2 . The amplified neural activity was integrated by a voltage integrator with a time constant of $0.05 \mathrm{~s}$. The table position was monitored by a potentiometer to show the tilting angle as a DC voltage. ABP was measured using a pressure transducer (attached to the body). These signals were connected to an amplifier (AP-601G; Nihon-Koden, Tokyo, Japan). Heart rate was calculated from the arterial pulse via off-line analysis. 


\subsection{Data analysis}

ABP signals, nerve activity, integrated nerve activity, $\mathrm{CBF}$ and tilt angle were saved on DAT tape (TEAC RX832; Tokyo, Japan) for off-line analysis. The signal traces were digitized at $2 \mathrm{kHz}$ with an analog-todigital converter (Interface Corp, PCI-3153; Hiroshima, Japan) via a low-pass filter with a cutoff of $8 \mathrm{kHz}$ and subsequently analyzed with MATLAB (MathWorks, Natick, MA, USA). The mean ABP was calculated as the diastolic pressure plus one-third of the pulse pressure for each beat, and the values are shown in Table 1 . Nerve activity was calculated as the area from the baseline and integrated traces every $1 \mathrm{~s}$. The mean $\mathrm{ABP}, \mathrm{CBF}$ and nerve activity data were averaged over one-second intervals during each HDR.

Analysis of variance with Dunnett's multiple comparison test was used to determine statistical significance of the experimental data. Student's $t$-test was used when comparing two groups. Differences were considered statistically significant at $P<0.05$.

\section{Results}

\subsection{Changes in $A B P$ during $H D T$ positioning}

Table 1 shows the mean ABP before and after HDR in anesthetized rats. The mean ABP values in the horizontal prone position in Experiments 1, 2, 3 and 4 were $95 \pm 5 \mathrm{~mm} \mathrm{Hg}$ (mean \pm SE), $94 \pm 3 \mathrm{~mm} \mathrm{Hg}, 100$ $\pm 7 \mathrm{~mm} \mathrm{Hg}$, and $88 \pm 4 \mathrm{~mm} \mathrm{Hg}$, respectively (Table 1). The mean ABP began to decrease during HDR (Figs. 1A, 2A and B, 3A and B, 4A, 5A and 6A). As shown in Table 1 , the pressure reached its lowest level approximately $7.5 \mathrm{~s}$ after the onset of HDR, after which the ABP recovered to the pre-HDR level within $1 \mathrm{~min}$ (Fig. 1A). The average mean ABP for all 32 trials was $95 \pm 3 \mathrm{~mm} \mathrm{Hg}$. The average reduction in $\mathrm{ABP}$ was $8 \pm 3 \mathrm{~mm} \mathrm{Hg}$, and the time from the onset of HDR to the maximum reduction in mean $\mathrm{ABP}$ was $7.3 \pm 0.3 \mathrm{~s}$ for all trials.

\subsection{Effects of hexamethonium and $P B$ on $A B P$}

The possibility that the decrease in ABP occurred through a mechanism that did not involve the autonomic nerves was next tested. In Experiment 1, four out of 8 rats were injected with hexamethonium, after which RSNA immediately and markedly decreased, as shown in Fig. 1B. The mean ABP significantly decreased from $93 \pm 7$ to $66 \pm 4 \mathrm{~mm} \mathrm{Hg}$ (n $=4, P<0.01$ ). HDR induced a significant decrease in mean ABP before hexamethonium injection, but this effect was not observed 2 min after the injection, as shown in Fig. 1. The average HDR-elicited decrease in

Table 1

Changes in arterial blood pressure during HDR.

\begin{tabular}{|c|c|c|c|c|}
\hline & \multicolumn{3}{|c|}{ Mean ABP (mm Hg) } & \multirow{2}{*}{$\begin{array}{l}\text { Latency of } \\
\text { maximum drop } \\
(\mathrm{s})^{\mathrm{d}}\end{array}$} \\
\hline & $\begin{array}{l}\text { Before } \\
\text { HDR } \\
\text { onset }^{\mathrm{a}}\end{array}$ & $\begin{array}{l}7.5 \mathrm{~s} \text { after } \\
\text { HDR onset }\end{array}$ & $\begin{array}{l}1 \text { min after } \\
\text { HDR onset }^{\mathrm{C}}\end{array}$ & \\
\hline $\begin{array}{l}\text { Experiment } 1 \text { ( } \mathrm{n} \\
\quad=8,8 \text { trials) }\end{array}$ & $95 \pm 5$ & $84 \pm 7^{*}$ & $95 \pm 6$ & $7.5 \pm 0.5$ \\
\hline $\begin{array}{l}\text { Experiment } 2 \text { (n } \\
\quad=8,8 \text { trials) }\end{array}$ & $94 \pm 3$ & $86 \pm 3^{*}$ & $93 \pm 3$ & $7.1 \pm 0.3$ \\
\hline $\begin{array}{l}\text { Experiment } 3 \text { ( } \mathrm{n} \\
\quad=8,8 \text { trials) }\end{array}$ & $100 \pm 7$ & $90 \pm 8^{*}$ & $99 \pm 7$ & $7.5 \pm 0.4$ \\
\hline $\begin{array}{l}\text { Experiment } 4 \text { (n } \\
\quad=8,8 \text { trials) }\end{array}$ & $88 \pm 4$ & $80 \pm 3^{*}$ & $88 \pm 4$ & $7 \pm 0.4$ \\
\hline
\end{tabular}

Asterisks indicate $P<0.05$ versus mean ABP before HDR.

a Average mean arterial blood pressure (ABP) for 1 to $2 \mathrm{~s}$ before onset of HDR for each trial.

b Average mean ABP at the head-down position for 7 to $8 \mathrm{~s}$ after onset of HDR.

c Average mean ABP at the head-down position for 59 to $60 \mathrm{~s}$ after onset of HDR.

d Time to maximum reduction of mean ABP.

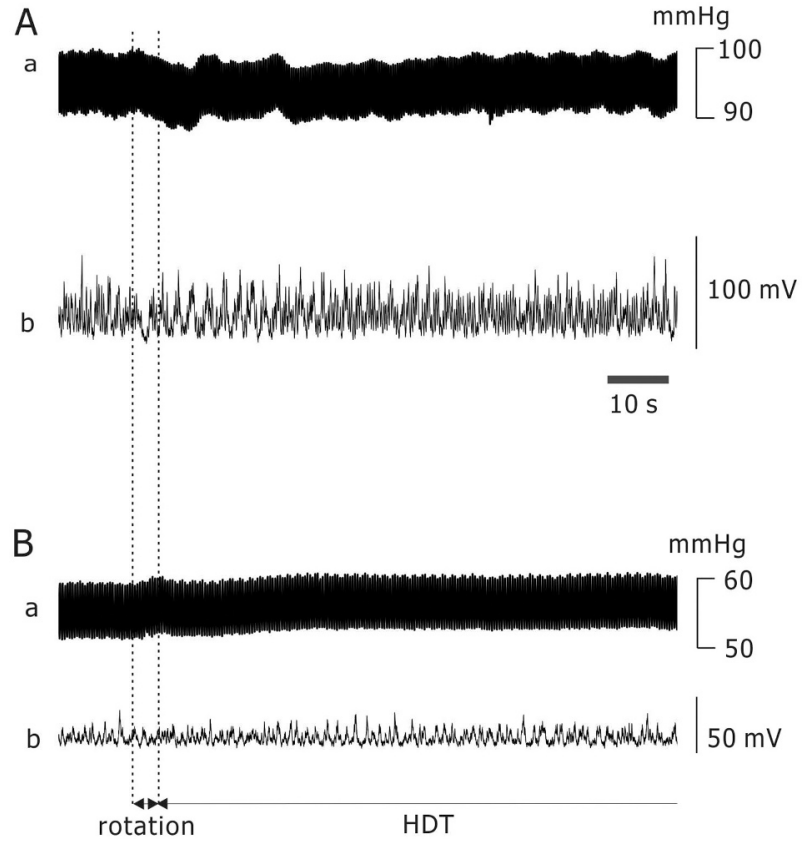

Fig. 1. Effect of pretreatment with hexamethonium on ABP and RSNA during HDR. A Traces show ABP (a) and integrated RSNA (b) of the control before the injection of hexamethonium. B Traces show ABP (a) and integrated RSNA (b) at 2 min after the injection of hexamethonium. Vertical dotted lines indicate the onset and end of rotation. HDT stands for head-down tilt.

mean $\mathrm{ABP}$ was significantly greater before injection $(7 \pm 3 \mathrm{~mm} \mathrm{Hg})$ than after injection $(-3 \pm 1 \mathrm{~mm} \mathrm{Hg})(\mathrm{n}=4, P<0.01$; not illustrated).

In Experiment 3, changes in $\mathrm{ABP}$ during $\mathrm{HDT}$ positioning were measured before and after intravenous administration of $\mathrm{PB}$, an $\alpha$-adrenergic blocker. The administration of PB induced a significant decrease in mean $\mathrm{ABP}$ from $100 \pm 8$ to $69 \pm 3 \mathrm{~mm} \mathrm{Hg}(\mathrm{n}=8, P<0.05)$. After administration, the ABP did not change significantly during or after HDR (Figs. 4B and 5B).

\subsection{Changes in RSNA and CSTA during HDT positioning}

Fig. 2 shows the typical changes in ABP, RSNA (Fig. 2A), and CSTA (Fig. 2B) observed in control rats. Sympathetic activity was identified as phasic heartbeat-related firing that corresponds to the descending phase in ABP (Kobayashi et al., 1999). HDR induced transient suppression of RSNA (Figs. 2Ab, c and 3C), which was followed by a transient decrease in ABP (Figs. 1Aa, 2Aa and 3A). The mean latency of the peak suppression from the onset of HDR was $2.3 \pm 0.4 \mathrm{~s}$ in the rats $(n=8,8$ trials). The peak suppression of RSNA preceded the initiation of the decrease in ABP, as shown in Fig. 3A and C. The heart rate did not change significantly during HDR (Table 2). These results suggest that the decrease in ABP was produced by the inhibition of sympathetic vasoconstrictor nerves. CSTA did not change significantly during HDR in the rats (Figs. 2Bb, c and 3D).

\subsection{Effects of HDR on CBF before and after the administration of an $\alpha$-blocker}

Fig. 4 shows the typical responses of ABP and CBF to HDR before (Fig. 4A) and after (Fig. 4B) intravenous administration of PB to a control rat. Intravenous injection of $\mathrm{PB}$ produced a significant decrease in the mean $\mathrm{ABP}$ from $100 \pm 7 \mathrm{~mm} \mathrm{Hg}$ to $66 \pm 4 \mathrm{~mm} \mathrm{Hg}(\mathrm{n}=8, P<$ 0.05). Before the injection, HDR induced a transient reduction in the mean ABP (Experiment 3 in Table 1, Figs. 4Aa and 5A); however, after the injection, HDR did not induce a significant reduction in ABP (Figs. 4Ba and 5B). The average CBF value at baseline before HDR was 

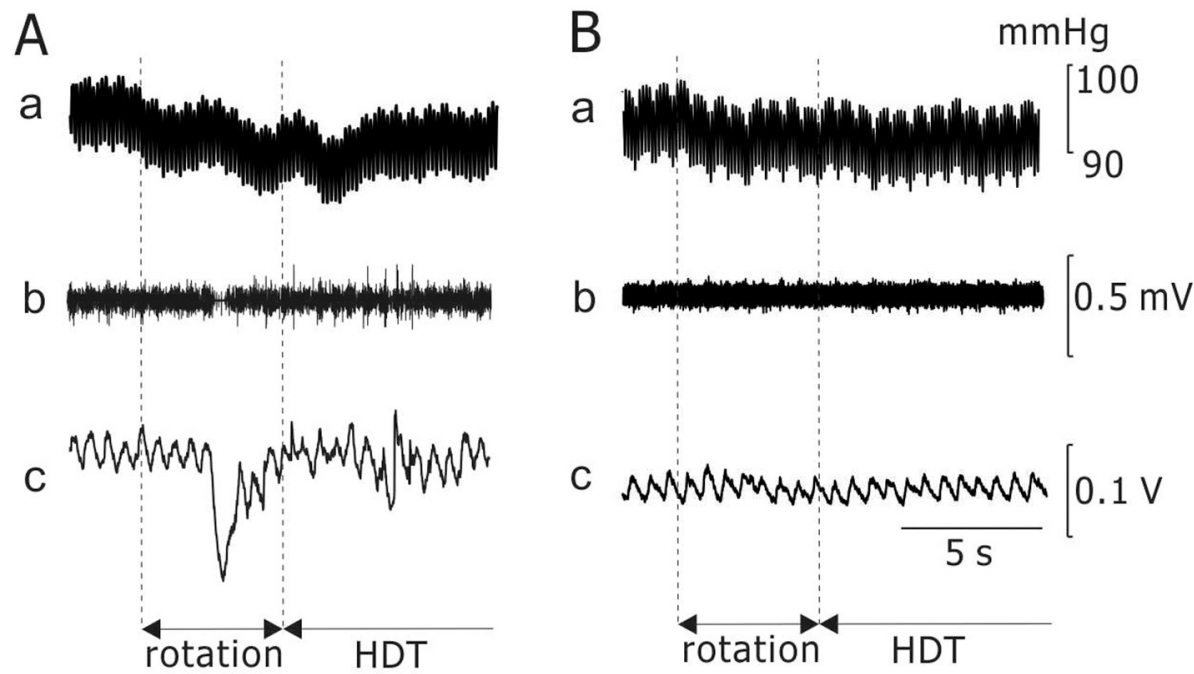

Fig. 2. Changes in ABP, RSNA, and CSTA during HDR.

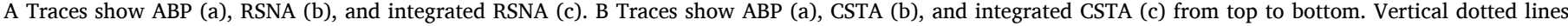
indicate the onset and end of rotation. HDT stands for head-down tilt.

A

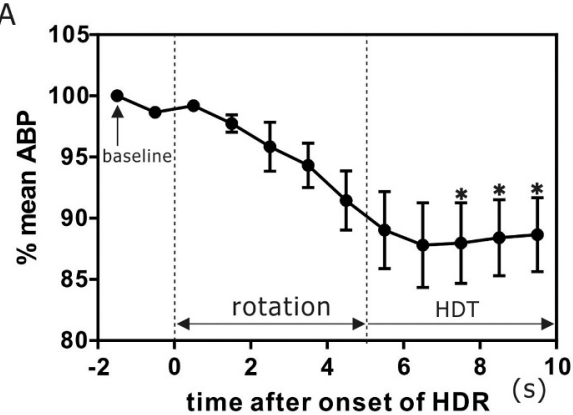

C

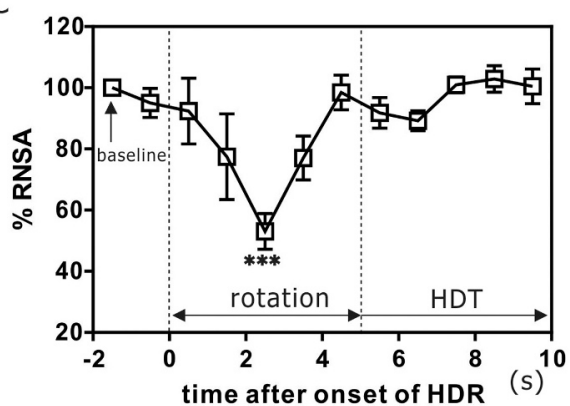

B

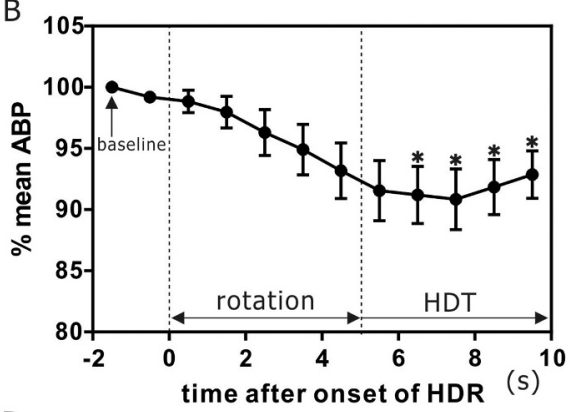

D

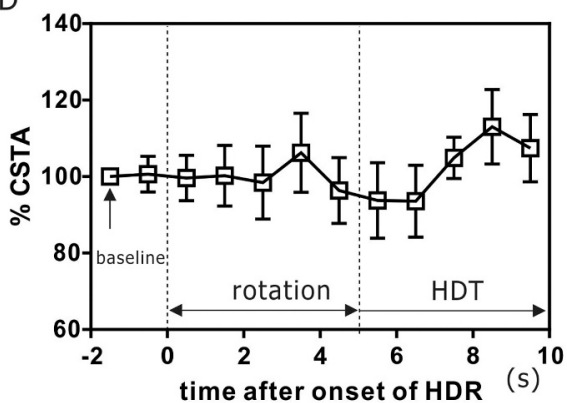

Fig. 3. Changes in mean ABP, RSNA, and CSTA during HDR.

Panels show summaries of changes in the mean ABP, RSNA, and CSTA with a one-second resolution. The left panels show the normalized mean ABP (A) and normalized RSNA (C) over time in Experiment 1. The right panels show the normalized mean $\mathrm{ABP}$ (B) and CSTA (D) over time in Experiment 2. Vertical dotted lines indicate the onset and the end of rotation. HDT stands for head-down tilt. Asterisks (*) indicate $P<$ 0.05 vs. baseline levels. Asterisks $(* * *)$ indicate $P<$ 0.0003 vs. baseline levels.
$1.9 \pm 0.3 \mathrm{ml} / \mathrm{min} / 100 \mathrm{~g}$ in control rats $(\mathrm{n}=8)$. As shown in Fig. 5C, HDR had a tendency to induce a small, transient reduction in CBF for $1.5 \mathrm{~s}$ and $2.5 \mathrm{~s}$ after the onset of HDR; however, these changes were not significant $(P=0.09)$. After the end of rotation, CBF did not differ significantly compared to that at baseline (Fig. 5C). Fig. 5D shows the changes in $\mathrm{CBF}$ during HDR after the administration of PB. Mean CBF after PB injection was $2.0 \pm 0.2 \mathrm{ml} / \mathrm{min} / 100 \mathrm{~g}$, which was not significantly different from that before the injection. HDR did not induce a significant change in CBF (Fig. 5D).

\subsection{Effects of HDR on CBF after bilateral denervation of the CSTs}

Fig. 6 shows the changes in ABP (Fig. 6A) and CBF (Fig. 6B) observed during HDR after bilateral denervation of the CSTs. The average mean ABP before denervation ( $94 \pm 4 \mathrm{~mm} \mathrm{Hg}, \mathrm{n}=8$ ) was not significantly different from that after denervation $(88 \pm 4 \mathrm{~mm} \mathrm{Hg})$. In addition, CBF before $(2.1 \pm 0.2 \mathrm{ml} / \mathrm{min} / 100 \mathrm{~g}, \mathrm{n}=8)$ or after denervation $(2.0 \pm 0.2$ $\mathrm{ml} / \mathrm{min} / 100 \mathrm{~g}, \mathrm{n}=8$ ) did not differ (not illustrated). HDR did not elicit a significant change in CBF (Fig. 6B), although HDR induced a transient reduction in the mean ABP (Experiment 4 in Table 1 and Fig. 6A).

\section{Discussion}

To summarize the major findings of this study, four points are of the greatest importance. 1) HDR induced transient suppression of RSNA; however, CSTA was not significantly changed. 2) ABP decreased transiently after the suppression of RSNA. 3) The HDR-elicited decrease in ABP disappeared after the administration of a ganglionic blocker and an $\alpha$-receptor blocker but did not disappear after denervation of the CSTs. 4) CBF did not change significantly during HDR in the control, after administration of an $\alpha$-receptor blocker, or after denervation of the CSTs. 


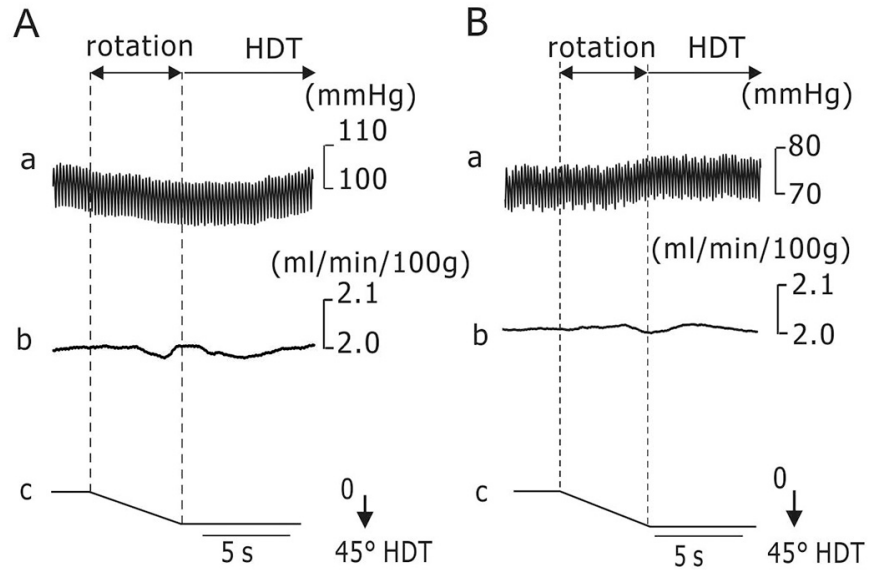

Fig. 4. Changes in $\mathrm{ABP}$ and $\mathrm{CBF}$ during HDR. A The left panel shows changes in $A B P(a), C B F(b)$, and the body position of the animal (c). B The right panel shows changes in ABP (a), CBF (b), and the body position of the animal (c) after intravenous injection of $\mathrm{PB}$. Vertical dotted lines indicate the onset and end of rotation. HDT stands for head-down tilt.

\subsection{Difference between RSNA and CSTA during HDR}

Changes in ABP during postural alterations are well known to be related to vestibular inputs (Uchino et al., 1970; Doba and Reis, 1974; Yates and Miller, 1994; Kerman and Yates, 1998; Wilson et al., 2006). In our previous studies, HDR was shown to elicit the transient suppression of RSNA, followed by a decrease in ABP after the end of HDR. Pretreatment with a ganglion-blocking agent, hexamethonium, eliminated both the suppression of RSNA and decrease in ABP by HDR (Nakamura et al., 2009). This study also showed that HDR induced the transient suppression of RSNA and that ABP decreased transiently after the suppression of RSNA, which is consistent with the data from the past study. BRs are well known to be elicited by fluid shifts, which are induced by postural alterations (Nagaya et al., 1995; Tanaka et al., 1999; Nakamura et al., 2009). This suppression is a more rapid response than BRs and may be induced by VSRs, because vestibular lesions were found to extinguish this more rapid response (Nakamura et al., 2009). The mean latency ( $2.3 \pm 0.4 \mathrm{~s})$ of the suppression of RSNA after HDR onset in this study was also quicker than the mean latency $(7.3 \pm 0.3 \mathrm{~s})$ of the decrease in ABP after the onset of HDR, suggesting that RSNA suppression is more rapid than BRs. HDR did not significantly change heart rate in the rats (Table 2). Therefore, the enhancement of cardiac vagal tone does not seem to play an important role in decreased ABP (Nakamura et al., 2009). Since the activity of the vestibular nerve was demonstrated to inhibit RSNA (Uchino et al., 1970; Kerman and Yates, 1998), HDR probably stimulates the vestibular nerves, which suppresses sympathetic outflow to the lower body. This sympathetic suppression might be a compensation mechanism against the rapid increase in CBF due to HDR-elicited cephalad fluid shift. Importantly, CSTA was not inhibited by HDR in this study. Kerman and Yates (1998) demonstrate that vestibular inputs affect the activity of sympathetic outflow with regional differences. Interestingly, the vestibulo-sympathetic suppression of renal sympathetic nerves is much greater than that of cervical sympathetic nerves (Kerman and Yates, 1998). This result indicates that vestibular stimulation hardly suppresses the activity of cervical sympathetic nerves, which is probably why CSTA did not show any changes during HDR. Another possibility is that CSTA did not change during HDR because of the use of urethane anesthesia, which increases ABP, heart rate, and RSNA (Shimokawa et al., 1998). The authors of a previous study demonstrated that urethane decreased the gain of BR control of heart rate but elicited no change in that of RSNA (Shimokawa et al., 1998.); therefore, CSTA might induce less change than RSNA during HDR. Further research using alert animals is needed to determine the effect of anesthetics.

There are limitations to our methods. As shown in Fig. 3A and C, RSNA was restored to the baseline level after $5 \mathrm{~s}$ of rotation but the blood pressure remained reduced, which showed that the reduction in blood pressure was not associated with RNSA recovery. BRs may restrict the increase in blood pressure when the HDT position is adopted (Nagaya et al., 1995; Tanaka et al., 1999); however, it is difficult to explain the dissociation by only the pattern of sympathetic nerve activity. Sympathetic and parasympathetic nerve activities were noted to show not only reciprocal but also nonreciprocal, co-activation or co-inhibition patterns (Paton et al., 2005). The authors of this study reported that many
A

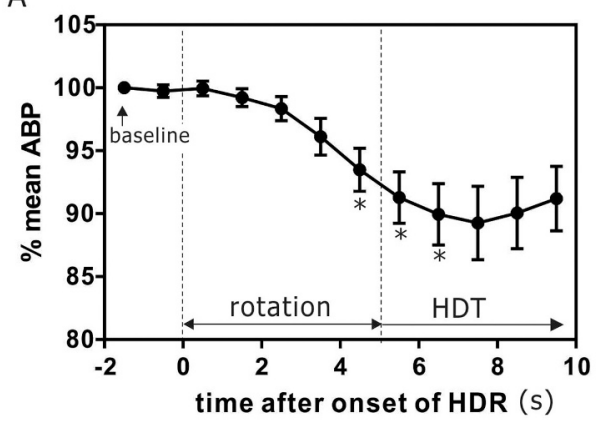

C

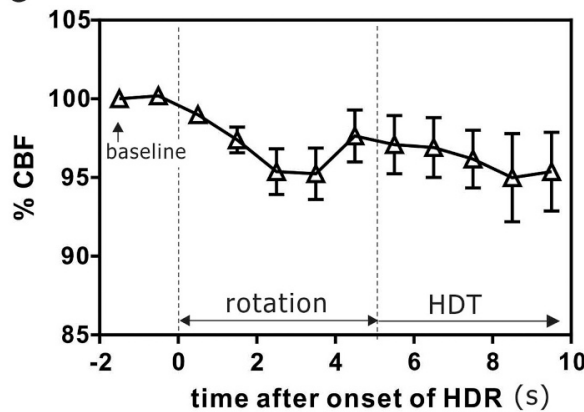

$\mathrm{B}$

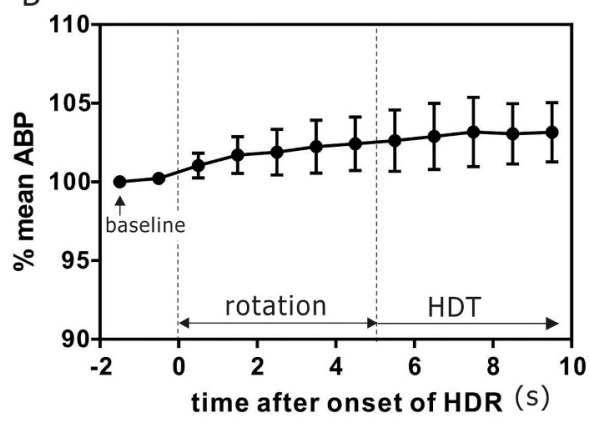

D

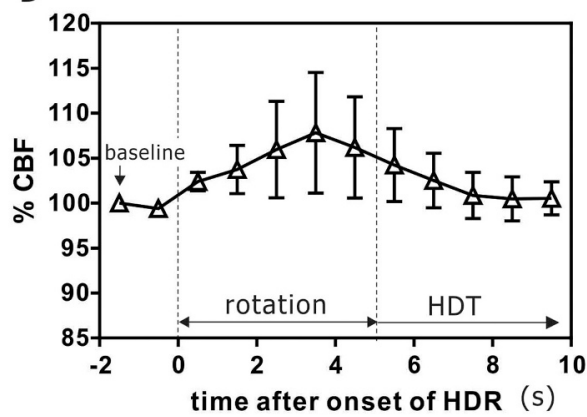

Fig. 5. Summary of changes in ABP and CBF during HDR in Experiment 3.

The left panels show the normalized mean ABP (A) and normalized $\mathrm{CBF}$ (C) over time. The right panels show the time course of the normalized mean ABP (B) and normalized $\mathrm{CBF}$ (D) over time after intravenous injection of PB. Vertical dotted lines indicate the onset and end of rotation. HDT stands for head-down tilt. Asterisks indicate $P<0.05$ vs. baseline levels. 

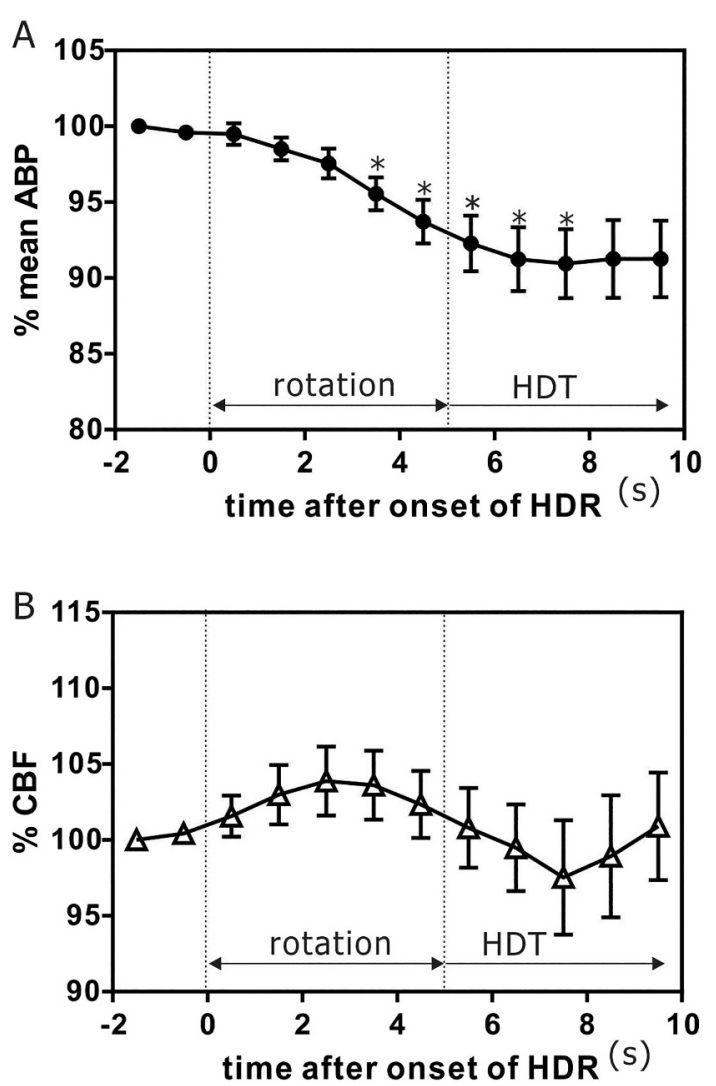

Fig. 6. Changes in mean $\mathrm{ABP}$ and $\mathrm{CBF}$ during HDR after denervation of the CSTs.

The top panel shows changes in the normalized mean ABP (A), and the bottom panel shows changes in the normalized CBF (B) after the CSTs were bilaterally cut. Vertical dotted lines indicate the onset and end of rotation. HDT stands for head-down tilt. Asterisks indicate $P<0.05$ vs. baseline levels

Table 2

Effect of HDR on heart rate in rats.

\begin{tabular}{llll}
\hline & Heart rate, bpm & & \\
\cline { 2 - 4 } & Before HDR & During HDR & After HDR \\
\hline Rats $(\mathrm{n}=32,32$ trials) & $380 \pm 8$ & $379 \pm 8$ & $377 \pm 8$ \\
\hline
\end{tabular}

Mean value of heart rate for $2 \mathrm{~s}$ before onset of HDR, during HDR, and after HDR.

protective reflexes, such as peripheral chemoreceptor, startle, noxious, ocular, and defensive reflexes, appear to simultaneously excite both autonomic outflows to the heart (Paton et al., 2005). Therefore, cardiovascular responses to co-activation of both the sympathetic and parasympathetic nerves might have overlapped in this case. The interpretation of results obtained by recording cardiac nerves is not simple (Paton et al., 2005). Moreover, the interactions of parasympathetic and sympathetic postganglionic neurons with the brain and systemic circulation are still unknown (Paton et al., 2005; Oliveira-Sales et al., 2016; Roloff et al., 2016). To clarify the mechanism of these sympathetic and parasympathetic interactions, further study using new methods is needed (Paton, 1996; Paton et al., 2005; Oliveira-Sales et al., 2016).

\subsection{Role of sympathetic nerve activity in $C B F$ during $H D R$}

As shown in Fig. 5, HDR tended to induce a small, transient reduction in $\mathrm{CBF}$ in the control during rotation; however, this change was not significant $(P=0.09)$. Moreover, CBF did not change significantly during HDR after the administration of an $\alpha$-receptor blocker or denervation of the CSTs in this study. These results suggest that the impact of the CSTs on CBF is probably limited by the increase in CBF due to a HDRelicited cephalad fluid shift. However, Gotoh et al. (1971) demonstrated that the loss of $\mathrm{CBF}$ autoregulation in patients with severe impairment of the autonomic nervous system during head-up tilt, such as that in ShyDrager syndrome. Interestingly, they showed that the degree of impairment of autoregulation was dependent on the extent of autonomic nervous system involvement, suggesting that changes in CBF during postural alterations might be affected by autonomic outflow (Gotoh et al., 1971).

It is clear that the CST innervates the cerebral artery (Nielsen and Owman, 1967; Iwayama et al., 1970; Edvinsson et al., 1975). Stimulation of the superior cervical ganglion has been proposed to induce a reduction in $\mathrm{CBF}$ at baseline as well as during acute hypertension (Heistad et al., 1978; Cassaglia et al., 2008). Some investigations in rats demonstrated that high-frequency $(20-30 \mathrm{~Hz})$ stimulation of CST induced a short-term increase in CBF, followed by a decrease in CBF (Saeki et al., 1990; Shiba et al., 2009). These results suggest that stimulation of the CST affects CBF in rats. Saeki et al. showed that the stimulation-induced decrease in CBF was abolished by an $\alpha$-adrenergic blocking agent (Saeki et al., 1990; Shiba et al., 2009). As shown in Fig. 5C, HDR had the tendency to induce a small transient reduction in CBF during HDR, which might be explained by the suppression of $\alpha$-adrenergic vascular tone; however, the change in CBF was not significant $(P=0.09)$. This result suggests that sympathetic outflow is unlikely to contribute in a major way to CBF autoregulation. However, there are limitations to our study as studies have also shown the role of parasympathetic involvement in the autoregulation of CBF (Kurosawa et al., 1989; Morita et al., 1994; Cipolla, 2009; Roloff et al., 2016). According to a report by Morita et al. (1994), although bilateral parasympathetic denervation did not affect either the frequency or amplitude of vasomotion, the lower limit of cerebral autoregulation was shifted towards a higher blood pressure in denervated rats (Morita et al., 1994), which suggests the contribution of parasympathetic nerves to cerebral autoregulation. Since focal electrical stimulation of the nucleus basalis of Meynert was reported to produce an increase in acetylcholine release and blood flow in the parietal cortex in rats (Kurosawa et al., 1989), intracranial basal forebrain cholinergic nerves should also be considered. Thus, further study is needed to clarify the influence of other neural systems that control CBF, including parasympathetic nerves and intracranial basal forebrain cholinergic nerves.

In terms of the changes in CBF observed during HDR, CBF in the control did not significantly change during HDR in this study. The change in CBF after bilateral denervation of the CSTs (Fig. 6B) and after the injection of PB (Fig. 5D) varied more widely than that in the control, but the flow was not significantly changed during HDR. According to a report by Sadoshima et al. (1981), superior cervical ganglionectomy did not affect CBF, although sympathetic nerve traffic increased fivefold during intracranial hypertension. They concluded that intense reflex activation of the sympathetic nerves during intracranial hypertension does not affect CBF (Sadoshima et al., 1981). These results suggest that an alternative mechanism for cerebral autoregulation other than a mechanism involving CSTA should be considered. Cipolla reported that autoregulation of CBF was most likely due to the myogenic behavior of cerebral smooth muscle when pressure fluctuated at the high end of the autoregulatory curve (Cipolla, 2009). Thus, our results during HDR can be explained by the contribution of myogenic activity to autoregulation, although involvement of the parasympathetic nerves is possible.

In conclusion, HDR suppressed RSNA but not CSTA in this study. The HDR-elicited suppression of sympathetic outflow to the lower body is probably larger than that to the head, which probably reduces systemic blood pressure. The consequent decrease in ABP reduces the CPP, which is the pressure gradient between the systemic ABP and ICP (Gierthmühlen et al., 2010). HDR did not change CSTA during rotation in this study. Furthermore, CBF did not change significantly during HDR. Denervation of the CSTs or pretreatment with an $\alpha$-blocker did not significantly affect CBF during HDR. Hence, changes in CBF may be 
elicited mainly by another mechanism, i.e., the autoregulation of cerebral vessels, which acts to keep CBF constant during HDR (Gierthmühlen et al., 2010; Ainslie and Brassard, 2014; Brassard et al., 2017), despite the possible contribution of CSTA to CBF regulation.

\section{Acknowledgments}

This study was supported by JSPS KAKENHI grant number JP 24592549.

\section{Declaration of competing interest}

The authors declare no conflicts of interest.

\section{References}

Ainslie, P.N., Brassard, P., 2014. Why is the neural control of cerebral autoregulation so controversial? F1000Prime Rep 6, 14. https://doi.org/10.12703/P6-14.

Asai, Y., Inoue, S., Tatebayashi, K., Shiraishi, Y., Kawai, Y., 2002. Effects of head-down tilt on cerebral blood flow and somatosensory-evoked potentials in rabbits. Jpn. J. Physiol. 52, 105-110. https://doi.org/10.2170/jjphysiol.52.105.

Brassard, P., Tymko, M.M., Ainslie, P.N., 2017. Sympathetic control of the brain circulation: appreciating the complexities to better understand the controversy. Auton. Neurosci. 207, 37-47. https://doi.org/10.1016/j.autneu.2017.05.003.

Cassaglia, P.A., Griffiths, R.I., Walker, A.M., 2008. Sympathetic nerve activity in the superior cervical ganglia increases in response to imposed increases in arterial pressure. Am. J. Physiol.-Regul. Integr. Comp. Physiol. 294, R1255-R1261. https:// doi.org/10.1152/ajpregu.00332.2007.

Cipolla, M.J., 2009. The Cerebral Circulation. Morgan \& Claypool Life Sciences, San Rafael.

DiBona, G.F., Sawin, L.L., 1985. Renal nerve activity in conscious rats during volume expansion and depletion. Am. J. Physiol.-Ren. Physiol. 248, F15-F23. https://doi. org/10.1152/ajprenal.1985.248.1.f15.

Doba, N., Reis, D.J., 1974. Role of the cerebellum and the vestibular apparatus in regulation of orthostatic reflexes in the cat. Circ. Res. 34, 9-18. https://doi.org/ 10.1161/01.res.34.1.9.

Eckberg, D.L., Abboud, F.M., Mark, A.L., 1976. Modulation of carotid baroreflex responsiveness in man: effects of posture and propranolol. J. Appl. Physiol. 41, 383-387. https://doi.org/10.1152/jappl.1976.41.3.383.

Edvinsson, L., Aubineau, P., Owman, C., Sercombe, R., Seylaz, J., 1975. Sympathetic innervation of cerebral arteries: prejunctional supersensitivity to norepinephrine after sympathectomy or cocaine treatment. Stroke 6, 525-530. https://doi.org/ 10.1161/01.str.6.5.525.

Gierthmühlen, J., Allardt, A., Sawade, M., Wasner, G., Baron, R., 2010. Role of sympathetic nervous system in activity-induced cerebral perfusion. J. Neurol. 257, 1798-1805. https://doi.org/10.1007/s00415-010-5613-5.

Gotoh, F., Ebihara, S.I., Toyoda, M., Shinohara, Y., 1971. Role of autonomic nervous system in autoregulation of human cerebral circulation. Eur. Neurol. 6, 203-207. https://doi.org/10.1159/000114493.

Guo, G.B., Thames, M.D., Abboud, F.M., 1982. Differential baroreflex control of heart rate and vascular resistance in rabbits. Relative role of carotid, aortic, and cardiopulmonary baroreceptors. Circ. Res. 50, 554-565. https://doi.org/10.1161/ 01.res.50.4.554.

Heistad, D.D., Marcus, M.L., Gross, P.M., 1978. Effects of sympathetic nerves on cerebral vessels in dog, cat, and monkey. Am. J. Physiol.-Heart Circ. Physiol. 235, H544-H552. https://doi.org/10.1152/ajpheart.1978.235.5.h544.

Iwayama, T., Furness, J.B., Burnstock, G., 1970. Dual adrenergic and cholinergic innervation of the cerebral arteries of the rat. Circ. Res. 26, 635-646. https://doi. org/10.1161/01.res.26.5.635.

Kawai, Y., Murthy, G., Watenpaugh, D.E., Breit, G.A., Deroshia, C.W., Hargens, A.R., 1993. Cerebral blood flow velocity in humans exposed to $24 \mathrm{~h}$ of head-down tilt. J. Appl. Physiol. 74, 3046-3051. https://doi.org/10.1152/jappl.1993.74.6.3046.

Kawai, Y., Doi, M., Matsuura, K., Setogawa, A., Hargens, A.R., Murthy, G., Ballard, R.E. Watenpaugh, D.E., 1997. Cerebral hemodynamics during simulated microgravity in humans and rats. In: Sharma, B.K., Takeda, N., Ganguly, N.K., Singal, P.K. (Eds.), Adaptation Biology and Medicine. Narosa Publishing House, New Delhi, pp. 155-162.

Kerman, I.A., Yates, B.J., 1998. Regional and functional differences in the distribution of vestibulosympathetic reflexes. Am. J. Physiol.-Regul. Integr. Comp. Physiol. 275, R824-R835. https://doi.org/10.1152/ajpregu.1998.275.3.r824.

Kobayashi, M., Cheng, Z.B., Tanaka, K., Nosaka, S., 1999. Is the aortic depressor nerve involved in arterial chemoreflexes in rats? J. Auton. Nerv. Syst. 78, 38-48. https:// doi.org/10.1016/s0165-1838(99)00054-5.

Kurosawa, M., Sato, A., Sato, Y., 1989. Well-maintained responses of acetylcholine release and blood flow in the cerebral cortex to focal electrical stimulation of the nucleus basalis of Meynert in aged rats. Neurosci. Lett. 100, 198-202. https://doi. org/10.1016/0304-3940(89)90684-8. van Lieshout, J.J., Secher, N.H., 2008. Point:counterpoint: sympathetic activity does/ does not influence cerebral blood flow. J. Appl. Physiol. 105, 1364-1366. https:// doi.org/10.1152/japplphysiol.90597.2008.

Low, P.A., Tomalia, V.A., 2015. Orthostatic hypotension: mechanisms, causes, management. J. Clin. Neurol. 11, 220-226. https://doi.org/10.3988/ jcn.2015.11.3.220.

Matsuo, S., Hosogai, M., Nakao, S., 1994. Ascending projections of posterior canalactivated excitatory and inhibitory secondary vestibular neurons to the mesodiencephalon in cats. Exp. Brain Res. 100, 7-17. https://doi.org/10.1007/ BF00227274.

Morita, Y., Hardebo, J.E., Bouskela, E., 1994. Influence of cerebrovascular parasympathetic nerves on resting cerebral blood flow, spontaneous vasomotion, autoregulation, hypercapnic vasodilation and sympathetic vasoconstriction. J. Auton. Nerv. Syst. 49 Suppl. S9-S14. https://doi.org/10.1016/0165-1838(94) 90079-5.

Murakami, M., Niwa, H., Kushikata, T., Watanabe, H., Hirota, K., Ono, K., Ohba, T. 2014. Inhalation anesthesia is preferable for recording rat cardiac function using an electrocardiogram. Biol. Pharm. Bull. 37, 834-839. https://doi.org/10.1248/bpb. b14-00012.

Nagaya, K., Wada, F., Nakamitsu, S., Sagawa, S., Shiraki, K., 1995. Responses of the circulatory system and muscle sympathetic nerve activity to head-down tilt in humans. Am. J. Physiol.-Regul. Integr. Comp. Physiol. 268, R1289-R1294. https:// doi.org/10.1152/ajpregu.1995.268.5.r1289.

Nakamura, Y., Matsuo, S., Hosogai, M., Kawai, Y., 2009. Vestibular control of arterial blood pressure during head-down postural change in anesthetized rabbits. Exp. Brain Res. 194, 563-570. https://doi.org/10.1007/s00221-009-1732-6.

Nielsen, K.C., Owman, C., 1967. Adrenergic innervation of pial arteries related to the circle of Willis in the cat. Brain Res. 6, 773-776. https://doi.org/10.1016/0006 8993(67)90134-5.

Oliveira-Sales, E.B., Colombari, E., Abdala, A.P., Campos, R.R., Paton, J.F., 2016 Sympathetic overactivity occurs before hypertension in the two-kidney, one-clip model. Exp. Physiol. 101 (1), 67-80. https://doi.org/10.1113/EP085390.

Paton, J.F., 1996. A working heart-brainstem preparation of the mouse. J. Neurosci. Methods 65 (1), 63-68. https://doi.org/10.1016/0165-0270(95)00147-6.

Paton, J.F., Boscan, P., Pickering, A.E., Nalivaiko, E., 2005. The yin and yang of cardiac autonomic control: vago-sympathetic interactions revisited. Brain Res. Rev. 49 (3), 555-565. https://doi.org/10.1016/j.brainresrev.2005.02.005.

Roloff, E.V., Tomiak-Baquero, A.M., Kasparov, S., Paton, J.F., 2016. Parasympathetic innervation of vertebrobasilar arteries: is this a potential clinical target? J. Physiol. 594 (22), 6463-6485. https://doi.org/10.1113/JP272450.

Sadoshima, S., Thames, M., Heistad, D., 1981. Cerebral blood flow during elevation of intracranial pressure: role of sympathetic nerves. Am. J. Physiol. 241(1), H78-H84. https://doi.org/10.1152/ajpheart.1981.241.1.H78.

Saeki, Y., Sato, A., Sato, Y., Trzebski, A., 1990. Effects of stimulation of cervical sympathetic trunks with various frequencies on the local cortical cerebral blood flow measured by laser doppler flowmetry in the rat. Jpn. J. Physiol. 40, 15-32. https:// doi.org/10.2170/jjphysiol.40.15.

Shiba, K., Machida, T., Uchida, S., Hotta, H., 2009. Sympathetic neural regulation of olfactory bulb blood flow in adult and aged rats. Auton. Neurosci. 147, 75-79. https://doi.org/10.1016/j.autneu.2009.01.007.

Shimokawa, A., Kunitake, T., Takasaki, M., Kannan, H., 1998. Differential effects of anesthetics on sympathetic nerve activity and arterial baroreceptor reflex in chronically instrumented rats. J. Auton. Nerv. Syst. 72 (1), 46-54. https://doi.org/ 10.1016/S0165-1838(98)00084-8.

Strandgaard, S., Sigurdsson, S.T., 2008. Counterpoint: sympathetic nerve activity does not influence cerebral blood flow. J. Appl. Physiol. 105, 1366-1367. https://doi.org/ 10.1152/japplphysiol.90597.2008a.

Tanaka, H., Davy, K.P., Seals, D.R., 1999. Cardiopulmonary baroreflex inhibition of sympathetic nerve activity is preserved with age in healthy humans. J. Physiol. 515, 249-254. https://doi.org/10.1111/j.1469-7793.1999.249ad.x.

Thames, M.D., Miller, B.D., Abboud, F.M., 1982. Baroreflex regulation of renal nerve activity during volume expansion. Am. J. Physiol.-Heart Circ. Physiol. 243, H810-H814. https://doi.org/10.1152/ajpheart.1982.243.5.h810.

Uchino, Y., Kudo, N., Tsuda, K., Iwamura, Y., 1970. Vestibular inhibition of sympathetic nerve activities. Brain Res. 22, 195-206. https://doi.org/10.1016/0006-8993(70) 90004-1.

Westerhof, B.E., Gisolf, J., Karemaker, J.M., Wesseling, K.H., Secher, N.H., van Lieshout, J.J., 2006. Time course analysis of baroreflex sensitivity during postural stress. Am. J. Physiol.-Heart Circ. Physiol. 291, H2864-H2874. https://doi.org/ 10.1152/ajpheart.01024.2005.

Willie, C.K., Tzeng, Y.C., Fisher, J.A., Ainslie, P.N., 2014. Integrative regulation of human brain blood flow. J. Physiol. 592 (5), 841-859. https://doi.org/10.1113/ jphysiol.2013.268953.

Wilson, T.D., Cotter, L.A., Draper, J.A., Misra, S.P., Rice, C.D., Cass, S.P., Yates, B.J., 2006. Effects of postural changes and removal of vestibular inputs on blood flow to the head of conscious felines. J. Appl. Physiol. 100, 1475-1482. https://doi.org/ 10.1152/japplphysiol.01585.2005.

Yates, B.J., Miller, A.D., 1994. Properties of sympathetic reflexes elicited by natural vestibular stimulation: implications for cardiovascular control. J. Neurophysiol. 71, 2087-2092. https://doi.org/10.1152/jn.1994.71.6.2087. 